\title{
Biomarkers in pancreatic adenocarcinoma: current perspectives
}

This article was published in the following Dove Press journal:

OncoTargets and Therapy

9 December 2016

Number of times this article has been viewed

\author{
Douglas S Swords \\ Matthew A Firpo \\ Courtney L Scaife \\ Sean J Mulvihill
}

Department of Surgery, University of Utah Health Sciences, Salt Lake City, UT, USA
Correspondence: Douglas S Swords University of Utah Health Sciences, Department of Surgery, 30 North 1900 East, Salt Lake City, UT 84I32, USA Tel + I 80 I 5878747

Fax + I $80 I 585$ I520

Email douglas.swords@hsc.utah.edu

\begin{abstract}
Pancreatic ductal adenocarcinoma (PDAC) has a poor prognosis, with a 5-year survival rate of $7.7 \%$. Most patients are diagnosed at an advanced stage not amenable to potentially curative resection. A substantial portion of this review is dedicated to reviewing the current literature on carbohydrate antigen (CA 19-9), which is currently the only guidelinerecommended biomarker for PDAC. It provides valuable prognostic information, can predict resectability, and is useful in decision making about neoadjuvant therapy. We also discuss carcinoembryonic antigen (CEA), CA 125, serum biomarker panels, circulating tumor cells, and cell-free nucleic acids. Although many biomarkers have now been studied in relation to PDAC, significant work still needs to be done to validate their usefulness in the early detection of PDAC and management of patients with PDAC.
\end{abstract}

Keywords: pancreatic cancer, biomarkers, screening, CA 19-9, CEA

\section{Introduction}

Despite making up only $3.1 \%$ of new cancer diagnoses, pancreatic ductal adenocarcinoma (PDAC) is currently the fourth leading cause of cancer-related death in the US, and has one of the worst outcomes of any malignancy, with a 5-year survival rate of $7.7 \% .{ }^{1}$ The National Cancer Institute estimates that 53,070 people will be diagnosed with pancreatic cancer and 41,780 patients will die of pancreatic cancer in $2016 .^{2}$ In contrast to most solid organ malignancies, in which there has been dramatic progress in recent years due to earlier diagnosis and targeted therapy, PDAC mortality rates are actually increasing. ${ }^{1}$ It is projected that by 2030 , pancreatic cancer will surpass breast, prostate, and colorectal cancers to become the second leading cause of cancer-related death, second only to lung cancer. ${ }^{3}$ Although only $10 \%-20 \%$ of patients are diagnosed at a stage amenable to resection, surgery remains the only potentially curative treatment for PDAC. Over the last 25 years, there have been significant advances in patient selection, surgical techniques, and the perioperative care of patients with PDAC. As a result, the morbidity and mortality of pancreas surgery have declined considerably. ${ }^{4-8}$ High-volume centers have reported 5-year survival rates as high as $27 \% .{ }^{9}$ For PDAC patients who undergo resection, the chances of further survival increase the longer the patients survive after resection, and a small subset of patients can experience long-term survival. ${ }^{10}$ For example, a recent study using data from the National Cancer Database (NCDB) from 1998 to 2002 showed that $3.9 \%$ of patients with resected PDAC lived for 10 years after diagnosis. ${ }^{11}$

The only biomarker currently recommended for clinical use by the National Comprehensive Cancer Network (NCCN) guidelines for PDAC is carbohydrate antigen 19-9 (CA 19-9). ${ }^{12}$ As such, a significant amount of this review is dedicated 
to review the available literature on the utility of this biomarker at several critical points in the clinical trajectory of PDAC patients with resectable disease. We discuss the sensitivity and specificity of CA 19-9, its ability to predict prognosis, and its utility in decision making about resectability and neoadjuvant therapy. We then discuss selected other serum antigen biomarkers as well as the potential role for panels of serum biomarkers which, when pooled together, can have much greater sensitivity and specificity than any single marker. We also discuss the case of human equilibrative nucleoside transporter 1 (hENT1) as an example of a mutation that could be used to guide targeted therapy. Finally, we discuss cell-free nucleic acid technologies and circulating tumor cells (CTCs). This review is not intended to be a comprehensive analysis of the multitude of biomarkers that have been evaluated for PDAC. Rather, it is intended to highlight the current clinical evidence for commonly used biomarkers, and to discuss technologies that we believe will be important in the coming years.

\section{Carbohydrate antigen 19-9}

CA 19-9, or sialyl Lewis antigen, is by far the most well-studied biomarker for PDAC, and the only one currently recommended for clinical use by the NCCN guidelines for PDAC. ${ }^{12}$ It is recommended that CA 19-9 be checked preoperatively in all patients with suspected PDAC being considered for surgery and/or neoadjuvant therapy, after resection prior to adjuvant therapy, and every 3-6 months for 2 years postoperatively. ${ }^{12}$ Despite this, national data suggest that CA $19-9$ is only measured in $25 \%$ of pancreas cancer patients. ${ }^{13}$ CA $19-9$ was first discovered in 1979 by using monoclonal antibodies in the serum of patients with advanced colorectal carcinoma, and later was found to be produced by pancreatic carcinoma. ${ }^{14,15}$ A commercial assay was developed in $1983 .{ }^{16} \mathrm{CA} 19-9$ has a number of mechanisms that may be involved in carcinogenesis. For example, induction of sialyl Lewis expression in cancer of digestive organs is accompanied by increased ability of cancer cells to adhere to endothelial cells through endothelial E-selectin. ${ }^{17}$ However, CA 19-9 is also secreted by normal biliary epithelium, and can be markedly elevated because of benign biliary stricture, extra-pancreatic malignancies that cause biliary obstruction, biliary infection (ie, cholangitis), and inflammatory processes (ie, pancreatitis). ${ }^{18,19} \mathrm{An}$ important limitation of CA $19-9$ is that $\sim 10 \%$ of the population do not generate the specific sialyl antigen and are thus termed nonsecretors. ${ }^{13,20,21}$

\section{Utility of CA 19-9 in screening in asymptomatic patients}

Two large studies have evaluated CA 19-9 in screening asymptomatic patients for possible PDAC. A study in Korea screened 70,940 asymptomatic patients using CA 19-9 and abdominal ultrasound. Of 1,063 cases who had a CA 19-9 level above the upper limit of normal, only 4 patients had pancreatic cancer and 11 other malignancies were found. ${ }^{22}$ The positive predictive value (PPV) was calculated at $0.9 \%$ in their asymptomatic population. A study in Japan screened 10,162 asymptomatic patients and found only $4(0.4 \%)$ cases of PDAC. ${ }^{23}$ Both of these studies concluded that screening asymptomatic individuals is not worthwhile for the early detection of PDAC.

\section{Sensitivity and specificity of CA 19-9 for PDAC in patients being evaluated for possible PDAC}

In 1990, Steinberg published a report summarizing 24 studies on the use of CA 19-9 in the diagnosis of PDAC. ${ }^{24}$ This study reported on a total of 1,040 patients and 3,282 controls. The mean estimates for sensitivity and specificity were $81 \%$ and $90 \%$, respectively, using an upper limit of normal level of $37-40 \mathrm{U} / \mathrm{mL} .{ }^{24}$ These sensitivity and specificity estimates have been often quoted in the literature since then. Notably, the controls in that study were healthy individuals without gastrointestinal complaints or known pancreatic abnormalities on imaging. In 2013, Poruk et al performed an updated meta-analysis to examine the sensitivity and specificity of CA 19-9 for PDAC using a control population of patients with known benign pancreatic disease. ${ }^{25} \mathrm{~A}$ total of 57 studies containing 3,285 patients with pancreatic cancer satisfied the selection criteria for sensitivity calculations, and 37 studies including 1,882 patients with benign pancreatic disease satisfied the selection criteria for specificity calculations. The mean sensitivity was $78.2 \%$ (95\% confidence interval [CI] $76.1 \%-80.2 \%)$, and the mean specificity was $82.8 \%(95 \%$ CI $79.9 \%-85.3 \%) .{ }^{25}$ The sensitivity estimate was similar to that reported by Steinberg. The lower specificity estimate likely better reflects the actual clinical use of CA 19-9 in discriminating benign and malignant conditions in patients presenting with periampullary disease. As a primary screening test, CA 19-9 is convenient and inexpensive, but its lack of specificity results in a large number of false-positive examinations requiring further investigation, limiting its practical applicability. 


\section{CA 19-9 as predictor of resectability}

Unresectable disease found only at laparotomy which was not detected by clinical staging remains a significant problem in PDAC. Such patients are subjected to the morbidity of a major surgery without deriving any therapeutic benefit. The reported negative predictive values (NPVs) of computed tomography $(\mathrm{CT})$ for predicting resectability range from $88.2 \%$ to $100 \%$, although most of these studies used older generation CT scanners. ${ }^{26-29}$ A recent study reported on 256 patients in the era of modern thin-slice CT scanners reported that $\mathrm{CT}$ predicted the absence of metastatic disease in $85 \%$ of patients. ${ }^{30}$ Several studies have examined whether CA 19-9 can augment imaging-based assessment of resectability. ${ }^{31-35}$ As an example, Maithel et al reviewed a database of 491 patients who underwent staging laparoscopy before planned pancreatic resection for PDAC. ${ }^{31}$ Median CA 19-9 was $131 \mathrm{U} / \mathrm{mL}$ for those who underwent resection versus $379 \mathrm{U} / \mathrm{mL}$ in those with unresectable disease $(P=0.003)$. A receiver operator characteristic (ROC) curve was developed for CA 19-9 and resectability, and the statistically optimal cutoff was $130 \mathrm{U} / \mathrm{mL}$. Unresectable disease was identified in $26 \%$ of those with CA $19-9>100 \mathrm{U} / \mathrm{mL}$ but only in $11 \%$ of those with CA $19-9<100 \mathrm{U} / \mathrm{mL}(P=0.003)$. CA 19-9 levels $>130 \mathrm{U} / \mathrm{mL}$ remained a predictor of unresectability on multivariate analysis. ${ }^{31}$ They suggested that CA 19-9 levels may allow surgeons to select patients for staging laparoscopy. Several other studies using a variety of cutoff points for CA 19-9 have shown similar findings. ${ }^{32-35}$

\section{CA 19-9 as a predictor of prognosis}

Numerous studies have demonstrated that elevated CA 19-9 levels are associated with worse stage-specific survival and recurrence. ${ }^{13,36-38}$ Postoperative CA 19-9 levels are thought to be a more accurate estimate of prognosis than those obtained prior to resection. ${ }^{36,37}$ Turrini et al showed that patients with elevated pretreatment CA 19-9 showed that patients with elevated pretreatment CA 19-9 whose CA 19-9 level normalized after surgery experienced comparable survival to those without pretreatment elevation. ${ }^{36}$ Hata et al demonstrated that CA 19-9 levels that remain elevated postoperatively are associated with positive margin status and early hepatic- and peritoneal-based recurrence. ${ }^{38}$ Sugiura et al performed an ROC analysis of CA 19-9 levels in 154 patients who underwent resection of PDAC..$^{39}$ They found that a CA 19-9 cutoff level of $100 \mathrm{U} / \mathrm{mL}$ was a significant predictor of recurrence within 6 months of surgery. Early recurrence occurred in 39 of 73 patients $(53 \%)$ with CA $19-9$ level $>100 \mathrm{U} / \mathrm{mL}$ but in only 9 of 81 patients (11\%) with a level $<100 \mathrm{U} / \mathrm{mL}$, and CA 19-9 remained a significant predictor of early recurrence on multivariate analysis (odds ratio [OR] 11.2). ${ }^{39}$

Several groups have examined the meaning of CA 19-9 level in patients treated with neoadjuvant therapy and patterns of response to neoadjuvant therapy. In a study from MD Anderson, Katz et al examined the ability of CA 19-9 level at time of diagnosis to predict successful completion of neoadjuvant therapy and surgical resection in patients with resectable disease. ${ }^{40}$ They found that CA 19-9 levels $>37 \mathrm{U} / \mathrm{mL}$ had a PPV for completing neoadjuvant therapy and resection of $86 \%$, but the NPV was only $33 \% .{ }^{40}$ Patients with borderline resectable disease who experienced a decrease of CA $19-9>50 \%$ during neoadjuvant therapy had higher odds or R0 margin status (OR 4.2, $P=0.05) .^{40}$ Finally, a decrease in CA 19-9 during neoadjuvant therapy was associated with improved overall survival (OS). ${ }^{40-42}$

Patients with undetectable CA 19-9 levels (nonsecretors) have survival that is superior to CA 19-9 secretors overall, but have equivalent stage-specific survival compared to normal-level $(<37 \mathrm{U} / \mathrm{mL})$ secretors. ${ }^{13,43}$ Brown et al evaluated the significance of CA 19-9 kinetics in an institutional database of 72 patients with at least 2 CA 19-9 levels planned for pancreatectomy. ${ }^{44}$ ROC analysis revealed that an absolute CA 19-9 increase of $\geq 50 \mathrm{U} / \mathrm{mL}$ and a rate of increase of $\geq 1 \mathrm{U} / \mathrm{mL}$ per day identified patients who were unresectable on exploration. An absolute increase of $<50 \mathrm{U} / \mathrm{mL}$ and a rate of increase of $<1 \mathrm{U} / \mathrm{mL}$ per day predicted improved OS in the overall cohort, but not in patients who were able to undergo resection. ${ }^{44}$

\section{Decision making about neoadjuvant therapy versus upfront surgery}

The NCCN guidelines state that neoadjuvant therapy should be considered in selected patients who appear technically resectable but have poor prognostic features such as very elevated CA 19-9, large tumor size, large regional lymph nodes, excessive weight loss, or extreme pain. ${ }^{12}$ A 2008 single-institution analysis of 143 patients failed to show cutoff level of CA 19-9 which would be useful to triage patients to neoadjuvant therapy, likely due to power issues. ${ }^{45}$ Bergquist et al recently used the NCDB to formally evaluate the impact of CA 19-9 elevation at time of diagnosis in 10,806 patients with anatomically resectable early-stage (Stage I and II) disease. ${ }^{13}$ They found that early-stage patients with CA 19-9 elevation had decreased survival at 1,2 , and 3 years (56\% versus $68 \%, 30 \%$ versus $42 \%$, and $15 \%$ versus $25 \%$; all $P<0.001)$ compared to those with normal levels. 
Notably, CA 19-9 nonsecretors had equivalent survival to normal-level patients. This effect of elevated CA 19-9 on mortality hazard remained statistically significant after adjusting for confounders (hazard ratio [HR] 1.26, 95\% CI 1.20-1.32). Neoadjuvant systemic chemotherapy followed by curative intent surgery was the only treatment that completely eliminated the survival disparity conferred by elevation of CA 19-9. The authors concluded that patients with elevated CA 19-9 at diagnosis should be considered "biologically borderline resectable" regardless of anatomic resectability based on imaging, and that neoadjuvant chemotherapy should be administered to all such patients. ${ }^{13}$

\section{Carcinoembryonic antigen}

Carcinoembryonic antigen (CEA) is the second most common serum biomarker currently used clinically to detect PDAC. Unlike CA 19-9, CEA is not recommended by the NCCN guidelines, although it is commonly obtained in clinical practice. The 2013 meta-analysis by Poruk et al estimated the mean sensitivity of CEA for detecting PDAC as $44.2 \%$ (95\% CI $38.5 \%-50.0 \%)$ and the mean specificity as $87.5 \%(95 \%$ CI $82.5 \%-91.2 \%) .{ }^{25}$ These findings are fairly similar to another recent review of 13 studies containing 1,323 cases, which reported a median sensitivity of $54 \%$ and a median specificity of $79 \% .{ }^{46}$ The fairly poor sensitivity in these studies indicates that CEA is inferior to CA 19-9 at identification of PDAC. However, the specificity is similar to CA 19-9, indicating that CEA performs well at identifying patients with benign disease. There is much less literature evaluating the prognostic impact of CEA compared to CA 19-9. Nonetheless, elevated CEA levels have been established as an independent predictor of decreased survival. ${ }^{47,48}$

\section{CA 125}

The utility of CA 19-9 is limited in patients with biliary obstruction, which elevates levels. CA 125 is a mucin-like transmembrane glycoprotein encoded by the MUC16 gene which is overexpressed on the surface of ovarian cancer cells and is also secreted by other cancer cell types. ${ }^{49,50}$ A recent study of 211 consecutive PDAC patients undergoing resection measured CA 19-9 and CA 125 within 14 days prior to surgery and evaluated them alongside other known prognostic factors. ${ }^{50}$ High preoperative CA 125, higher stage, and lymph node status were independent predictors of OS and recurrence-free survival (RFS) in all patients, including those with elevated bilirubin. CA 19-9 was not predictive in those with elevated bilirubin, and there was no relationship between CA 125 and bilirubin levels. In patients with normal bilirubin, CA 19-9 was more predictive of OS and RFS than CA $125 .{ }^{50}$ One study compared the utility of CA 125 in predicting resectability compared to 6 other biomarkers including CA 19-9. ${ }^{51}$ CA 125 was found to have a superior predictive ability at predicting resectability compared to CA 19-9 and the other tested biomarkers. ${ }^{51}$

\section{hENT I}

Gemcitabine (GEM) is a pyrimidine nucleoside drug with efficacy against PDAC which is taken into cells by a nucleoside transporter. ${ }^{52}$ hENT1 is a nucleoside transporter that may predict response to adjuvant GEM therapy. A retrospective study of tissue samples from the ESPAC-3 trial found that hENT1 expression predicted response to GEM but not 5-fluorouracil (5-FU). ${ }^{53}$ Median survival for patients treated with GEM was 17.1 months for those with low hENT1 expression versus 26.2 months for those with high hENT1 expression $(P=0.002)$. For the 5-FU group, median survival was 25.6 and 21.9 months for those with low and high hENT1 expression, respectively $(P=0.36)$. hENT1 expression remained a significant predictor of survival on multivariate analysis. ${ }^{53}$ Samples from the RTOG9704 trial were examined in a similar fashion, and hENT1 expression was associated with OS and disease-free survival in a multivariate model in the group given GEM but not the group given 5-FU. ${ }^{54}$ Notably, both of these studies were performed using immunohistochemistry (IHC) with the 10D7G2 antibody. Retrospective analyses of the adjuvant and AIOPK0104 CONKO-00 trials used a different antibody for the IHC analysis, and did not replicate these findings. ${ }^{55,56}$ Unfortunately, the 10D7G2 antibody is currently not commercially available, and its utility would be limited to those cases for which tumor tissue is available for analysis. hENT1 is the main transporter of fluorothymidine (FLT), and it is possible that positron emission tomography imaging of FLT transport may act as a surrogate indicator for response to GEM. This possibility has been evaluated in vitro, but requires in vivo validation. ${ }^{57}$

\section{Serum biomarker panels}

As discussed in the section about CA 19-9, screening asymptomatic populations is problematic because of the potential for large numbers of false-positive examination. This same issue applies to a lesser extent in screening highrisk populations. Several studies have evaluated panels of biomarkers as a way of developing an overall test with a much higher sensitivity and specificity than that of any currently 
available single biomarker. ${ }^{58-61}$ Brand et al performed one of the largest studies of biomarkers to date by screening for expression of 83 serum biomarkers in 333 patients with PDAC, 114 patients with benign pancreatic conditions, and 227 healthy controls. ${ }^{61}$ A cohort of 203 patients with breast, lung, or colon cancer was also included. They found that 42 of the 83 biomarkers differed significantly between PDAC patients and healthy and benign pancreatic disease controls. After splitting their cohort into validation and training sets, a Metropolis algorithm with Monte Carlo simulation was utilized to analyze all possible panels consisting of 2,3 , and 4 biomarkers. In their validation set, the panel of CA 19-9, intercellular adhesion molecule 1 (ICAM-1), and osteoprotegerin (OPG) demonstrated a sensitivity and a specificity of $78 \%$ and $94 \%$, respectively, while the panel of CA 19-9, CEA, and tissue inhibitor of metalloproteinase-1 (TIMP-1) demonstrated a sensitivity and a specificity of $71 \%$ and $89 \%$, respectively. The CA 19-9, ICAM-1, and OPG panel was selective for PDAC and did not recognize breast, lung, or colon cancers (all specificities $\geq 97 \%$ ). ${ }^{61}$ Similar results were seen by our group, which evaluated the panel of osteopontin, TIMP-1, and CA 19-9 achieving a sensitivity of $87 \%$, a specificity of $91 \%$, and an overall accuracy of $89.5 \% .{ }^{59}$ However, a follow-up to the Brand study utilizing pre-diagnostic serum samples from the Prostate, Lung, Colorectal, and Ovarian Screening Trial showed that the original panels were ineffective, perhaps illustrating a disadvantage of limiting panels to a minimal number of biomarkers. ${ }^{62}$

Firpo et al used a mathematical modeling approach to investigate the number of serum biomarkers that would be necessary to achieve a panel screen that could be practical in the general population, starting with the assumption that sensitivity would need to be $\geq 99 \%$ in order to raise the PPV to a clinically actionable level. ${ }^{63}$ They started by identifying 9 biomarkers that are known to have elevated levels in PDAC patients compared to controls. They determined that a panel consisting of 40 biomarkers characterized individually by $32 \%$ sensitivity at $95 \%$ specificity would require any 7 biomarkers to be above the threshold and would result in a panel sensitivity of at least $99 \% .{ }^{63}$ A recent multicenter study utilized a 293-plex antibody microarray to identify protein profiles associated with PDAC in a sample of 156 patients with PDAC, 152 with other pancreatic disease, and 30 controls with non-pancreatic diseases. ${ }^{64}$ They found that a biomarker signature could be identified using up to 10 biomarkers to differentiate PDAC from controls with $>90 \%$ sensitivity and specificity. However, the implications of these findings on the utility of this approach for screening were limited because no healthy control subjects were evaluated, comparing instead to non-pancreatic disease controls. ${ }^{64}$ More recently, the same group compared the signature of PDAC patients to healthy controls, and found similar results. Additionally, in this study, they found that accuracy of the signature increased with increasing stage. ${ }^{65}$ For screening, of course, detection of the earliest stage (IA) pancreatic cancer, or the presence of high-grade dysplasia in pancreatic intraepithelial neoplasia or intraductal pancreatic mucinous neoplasm is the relevant goal.

We are not aware of any published experience with using serum biomarker panels to screen the general population or high-risk individuals. Although a complete discussion of the role of screening is beyond the scope of this review, it is generally agreed upon that screening the general population is not advisable or cost-effective, due to the low prevalence of PDAC. Efforts for screening need to be focused on those with $>10$-fold increased risk of PDAC, who account for $\sim 10 \%$ of PDAC patients. One recently published study established that screening high-risk individuals can be successful. ${ }^{66}$ Vasen et al performed a screening program consisting of yearly magnetic resonance imaging and possible endoscopic ultrasound for patients with familial pancreatic cancer (FPC) or gene defects known to predispose to PDAC. They found that surveillance of $C D K N 2 A$ mutation carriers was relatively successful; PDAC was detected in 13 of $178(7.3 \%) C D K N 2 A$ mutation carriers. Of these $13 C D K N 2 A$ mutation carriers who developed PDAC, the resection rate was $75 \%$ and the 5 -year survival rate was $24 \%$. The program was less clearly beneficial in FPC patients. ${ }^{66}$ The success of this screening program for high-risk patients shows that screening programs can be successful at increasing detection at a resectable stage and improve survival. Although a multitude of biomarkers have been described in the context of PDAC in recent years, we are unaware of any published efforts of screening programs of high-risk patients which incorporate biomarker panels. A recent systematic review ranked the currently available PDAC biomarkers according to the Reporting Recommendation for tumor Marker Prognostic Studies (REMARK) scoring system. ${ }^{67}$ These results should be used to develop biomarker panels that can be applied to screening patients at high risk of PDAC.

\section{Cell-free nucleic acids MicroRNAs}

MicroRNAs (miRNAs) are endogenous noncoding RNAs of 19-25 nucleotides that negatively regulate gene expression posttranscriptionally by targeting mRNA for cleavage or 
translational repression, and they are estimated to regulate over $60 \%$ of human genes. ${ }^{68}$ miRNA dysregulation plays an important role in the cancer formation and progression, and miRNAs can act as tumor suppressors or oncogenes. ${ }^{69}$ miRNA expression patterns are significantly altered in PDAC, and several studies have identified signatures associated with diagnosis, stage, progression, survival, and response to specific chemotherapy agents. ${ }^{70-72}$ A recent study examined the ability of miRNAs to differentiate tissue from intraductal papillary mucinous neoplasms (IPMNs) and PDAC from controls. ${ }^{73}$ The authors identified 607 miRNAs that were significantly dysregulated in PDAC and 396 in IPMN using next-generation sequencing. Of these, 40 miRNAs were commonly overexpressed in both. They validated their results in two other cohorts, including one with tissue obtained during endoscopic ultrasound fine-needle aspiration (EUS-FNA). They validated 30 miRNAs that were dysregulated in both PDAC and IPMN compared to controls. Importantly, their work shows that detecting these miRNAs in samples obtained from EUS-FNA is feasible, making these good biomarker candidates for early detection of PDAC. ${ }^{73}$

\section{Cell-free DNA}

Cell-free DNA (cfDNA) exists in the circulation as small DNA fragments. cfDNA is derived from DNA released into the bloodstream after cellular necrosis or apoptosis. ${ }^{74}$ The detection of tumor-derived DNA in cfDNA, known as circulating tumor DNA (ctDNA), provides the opportunity to diagnose cancers and monitor chemotherapy-resistant mutations. ${ }^{75}$ Hadano et al recently used droplet digital polymerase chain reaction to detect rare mutant tumor-derived $K R A S$ genes in plasma cfDNA as ctDNA. ${ }^{76}$ Of 105 patients undergoing pancreaticoduodenectomy for PDAC, ctDNA tumor-derived KRAS mutations were detected in $31 \%$. OS was significantly poorer in patients with ctDNA (median OS 13.6 months versus 27.6 months, $P<0.001$ ). Presence of ctDNA remained a significant predictor of worse OS on multivariate analysis (HR 3.2, 95\% CI 1.8-5.4).$^{76}$ Another study evaluated ctDNA KRAS mutations in 14 patients with advanced pancreatic cancer. ${ }^{77}$ Ten (71\%) patients had ctDNA prior to starting chemotherapy. Pre-therapy ctDNA level was a significant predictor of progression-free survival $(P=0.014)$ and $\mathrm{OS}(P=0.010) .{ }^{77}$ While absence of cfDNA in patients with confirmed PDAC seems likely to be clinically useful in predicting improved prognosis, the utility of cfDNA in early detection is likely to be limited by the relatively small proportion of patients with detectable levels.

\section{Circulating tumor cells}

CTCs are neoplastic cells shed into the bloodstream by a solid tumor. ${ }^{78,79}$ CTCs are found frequently in the blood of patients with malignancies, but rarely in healthy controls, and are believed to be a source of distant metastases. ${ }^{80,81}$ CTCs have been found in the blood of patients with all stages of PDAC, and their presence has been shown to be associated with poorer survival in several studies. ${ }^{79,82-86}$ A recent study from Johns Hopkins Hospital evaluated blood samples from 50 patients with PDAC and tested for CTCs using immunofluorescence for cytokeratin, vimentin, and CD45. ${ }^{87}$ Most studies of CTCs in PDAC have defined CTCs by the epithelial marker cytokeratin. The recent Johns Hopkins study characterized PDAC CTCs using both a mesenchymal marker (vimentin) and epithelial markers (cytokeratin positive, CD45 negative), which is relevant because the "epithelial-to-mesenchymal transition" is thought to facilitate metastasis. ${ }^{88}$ The authors found cells with the epithelial phenotype (cytokeratin positive, CD45 negative) in 39 of 50 patients (78\%). Of those, 26 patients $(67 \%)$ had CTCs that were also vimentin positive, CD45 negative (the "mesenchymal-like" phenotype). On multivariate analysis, only presence of vimentin-positive CTCs was significantly associated with tumor recurrence (HR 2.78, 95\% CI 1.31-5.88). However, the presence of cytokeratin-positive CTCs $(P<0.001)$ but not mesenchymallike CTCs $(P=0.39)$ was associated with $\mathrm{OS}^{87}$

While CTCs are not found in the blood of healthy controls, their presence is not definitive for PDAC. A recent study evaluated the significance of circulating epithelial cells (CECs) in 179 patients with pancreatic lesions. ${ }^{89} \mathrm{CECs}$ were identified in $49 \%$ of patients with PDAC, $64 \%$ of patients with neuroendocrine tumors, $62 \%$ of patients with IPMNs, and in $46 \%$ of patients with chronic pancreatitis $(P=0.41)$. CECs were morphologically similar between patients with PDAC, and benign and premalignant lesions, and did not confer worse prognosis in those with PDAC. ${ }^{89}$ The utility of using the presence of CTCs as a diagnostic tool is diminished by their rarity in PDAC; they are only found in 50\% of cases, and are found even less often in early-stage cases. ${ }^{90}$ Consensus on appropriate phenotypic markers, including cell surface antigens and cell size, in the context of circulating leukocytes is lacking. The use of CTCs for identification of molecular targets holds promise.

\section{Conclusion}

PDAC continues to have one of the worst overall outcomes of any solid organ malignancy, and there has been little 
progress in recent years. There is currently an urgent need for validation of strategies using both novel and known biomarkers for early detection, diagnosis, prediction of prognosis, and stratification for different therapeutic approaches. CA 19-9 is useful for predicting prognosis and monitoring treatment. Patients with significant CA 19-9 elevation should be strongly considered for neoadjuvant therapy even if the tumor appears resectable on imaging. Those with elevated CA 19-9 preoperatively (regardless of whether neoadjuvant therapy was given) should be considered for staging laparoscopy prior to laparotomy. CEA elevations above the upper limit of normal are a poor prognostic sign. CA 125 should be considered for clinical decision making for patients who are nonsecretors of CA 19-9. Serum biomarker panels can achieve levels of sensitivity, specificity, and overall accuracy that are unlikely to be matched by any single biomarker. Strategies applying a blood-based biomarker panel to screen those at high risk of PDAC should be further investigated. miRNAs, cfDNAs, and CTCs are promising new technologies that are likely to play a major role in management of PDAC in coming years, but the utility of cfDNAs and CTCs is likely to be limited in screening and early detection by the small percent of patients who express these biomarkers. A multitude of biomarkers have been identified in PDAC in recent years. Prospective studies are needed to rigorously investigate the clinical impact of incorporating these biomarkers into clinical decision making in order to improve outcomes in this disease.

\section{Disclosure}

The authors report no conflicts of interest in this work.

\section{References}

1. Siegel RL, Miller KD, Jemal A. Cancer statistics, 2015. CA Cancer J Clin. 2015;65(1):5-29.

2. National Cancer Institute. SEER stat fact sheets: pancreas cancer. Available from: http://seer.cancer.gov/statfacts/html/pancreas.html. Accessed July 28, 2016.

3. Rahib L, Smith BD, Aizenberg R, Rosenzweig AB, Fleshman JM, Matrisian LM. Projecting cancer incidence and deaths to 2030: the unexpected burden of thyroid, liver, and pancreas cancers in the United States. Cancer Res. 2014;74(11):2913-2921.

4. Fernandez-del Castillo C, Rattner DW, Warshaw AL. Standards for pancreatic resection in the 1990s. Arch Surg. 1995;130(3):295-299; discussion 299-300.

5. Richter A, Niedergethmann M, Sturm JW, Lorenz D, Post S, Trede M. Long-term results of partial pancreaticoduodenectomy for ductal adenocarcinoma of the pancreatic head: 25-year experience. World J Surg. 2003;27(3):324-329.

6. Schmidt CM, Powell ES, Yiannoutsos CT, et al. Pancreaticoduodenectomy: a 20-year experience in 516 patients. Arch Surg. 2004;139(7):718-725; discussion 725-727.
7. Sohn TA, Yeo CJ, Cameron JL, et al. Resected adenocarcinoma of the pancreas-616 patients: results, outcomes, and prognostic indicators. J Gastrointest Surg. 2000;4(6):567-579.

8. Cameron JL, He J. Two thousand consecutive pancreaticoduodenectomies. J Am Coll Surg. 2015;220(4):530-536.

9. Katz MGH, Wang H, Fleming JB, et al. Long-term survival after multidisciplinary management of resected pancreatic adenocarcinoma. Ann Surg Oncol. 2009;16(4):836-847.

10. Mayo SC, Nathan H, Cameron JL, et al. Conditional survival in patients with pancreatic ductal adenocarcinoma resected with curative intent. Cancer. 2012;118(10):2674-2681.

11. Paniccia A, Hosokawa MS, Henderson W, et al. Characteristics of 10-year survivors of pancreatic ductal adenocarcinoma. JAMA Surg. 2015;150(8):701-710.

12. National Cancer Comprehensive Network. Pancreatic adenocarcinoma guidelines (version 1.2016). Available from: https://www.nccn.org/ professionals/physician_gls/PDF/pancreatic.pdf. Accessed July 28, 2016.

13. Bergquist JR, Puig CA, Shubert CR, et al. Carbohydrate antigen 19-9 elevation in anatomically resectable, early stage pancreatic cancer is independently associated with decreased overall survival and an indication for neoadjuvant therapy: a National Cancer Database study. J Am Coll Surg. 2016;223(1):52-65.

14. Koprowski H, Steplewski, Mitchell K, Herlyn M, Herlyn D, Fuhrer P. Colorectal carcinoma antigens detected by hybridoma antibodies. Somatic Cell Genet. 1979;5(6):957-971.

15. Koprowski H, Herlyn M, Steplewski Z, Sears HF. Specific antigen in serum of patients with colon carcinoma. Science. 1981;212(4490): 53-55.

16. Del Villano BC, Brennan S, Brock P, et al. Radioimmunometric assay for a monoclonal antibody-defined tumor marker, CA 19-9. Clin Chem. 1983;29(3):549-552.

17. Miyazaki K, Ohmori K, Izawa M, et al. Loss of disialyl Lewis(a), the ligand for lymphocyte inhibitory receptor sialic acid-binding immunoglobulin-like lectin-7 (Siglec-7) associated with increased sialyl Lewis(a) expression on human colon cancers. Cancer Res. 2004;64(13): 4498-4505.

18. Kannagi R. Carbohydrate antigen sialyl Lewis a - its pathophysiological significance and induction mechanism in cancer progression. Chang Gung Med J. 2007;30(3):189-209.

19. Scoggins CR, Moore D, Washington K, Wright JK, Chari RS. Supraelevated CA 19-9 in a benign hepatic cyst adenoma. HPB (Oxford). 2004;6(1):43-33.

20. Orntoft TF, Vestergaard EM, Holmes E, et al. Influence of Lewis alpha13/4-L-fucosyltransferase (FUT3) gene mutations on enzyme activity, erythrocyte phenotyping, and circulating tumor marker sialyl-Lewis a levels. J Biol Chem. 1996;271(50):32260-32268.

21. Yu LC, Yang YH, Broadberry RE, Chen YH, Chan YS, Lin M. Correlation of a missense mutation in the human Secretor alpha 1,2fucosyltransferase gene with the Lewis $(a+b+)$ phenotype: a potential molecular basis for the weak Secretor allele (Sew). Biochem J. 1995; 312(Pt 2):329-332.

22. Kim JE, Lee KT, Lee JK, Paik SW, Rhee JC, Choi KW. Clinical usefulness of carbohydrate antigen 19-9 as a screening test for pancreatic cancer in an asymptomatic population. J Gastroenterol Hepatol. 2004; 19(2):182-186.

23. Homma T, Tsuchiya R. The study of mass screening of persons without symptoms and of the screening of outpatients with gastrointestinal symptoms or icterus for pancreatic cancer in Japan, using CA19-9 and elastase-1 or ultrasonography. Int J Pancreatol. 1991;9:119-124.

24. Steinberg W. The clinical utility of CA 19-9 tumor-associated antigen. Am J Gastroenterol. 1990;85(4):350-355.

25. Poruk KE, Gay DZ, Brown K, et al. The clinical utility of CA 19-9 in pancreatic adenocarcinoma: diagnostic and prognostic updates. Curr Mol Med. 2013;13(3):340-351. 
26. Shah D, Fisher WE, Hodges SE, Wu MF, Hilsenbeck SG, Brunicardi CF. Preoperative prediction of complete resection in pancreatic cancer. J Surg Res. 2008;147(2):216-220.

27. Zamboni GA, Kruskal JB, Vollmer CM, Baptista J, Callery MP, Raptopoulos VD. Pancreatic adenocarcinoma: value of multidetector CT angiography in preoperative evaluation. Radiology. 2007;245(3): 770-778.

28. Karmazanovsky G, Fedorov V, Kubyshkin V, Kotchatkov A. Pancreatic head cancer: accuracy of $\mathrm{CT}$ in determination of resectability. Abdom Imaging. 2005;30(4):488-500.

29. Smith SL, Basu A, Rae DM, Sinclair M. Preoperative staging of multidetector computed tomography in pancreatic head adenocarcinoma. Pancreas. 2007;34(2):180-184.

30. Raman SP, Reddy S, Weiss MJ, et al. Impact of the time interval between MDCT imaging and surgery on the accuracy of identifying metastatic disease in patients with pancreatic cancer. AJR Am J Roentgenol. 2015; 204(1):W37-W42.

31. Maithel SK, Maloney S, Winston C, et al. Preoperative CA 19-9 and the yield of staging laparoscopy in patients with radiographically resectable pancreatic adenocarcinoma. Ann Surg Oncol. 2008;15(12): $3512-3520$.

32. Karachristos K, Scarmeas N, Hoffman JP. CA 19-9 levels predict results of staging laparoscopy in pancreatic cancer. J Gastrointest Surg. 2005;9(9):1286-1292.

33. Alexakis N, Gomatos IP, Sbarounis S, et al. High serum CA 19-9 but not tumor size should select patients for staging laparoscopy in radiological resectable pancreas head and peri-ampullary cancer. Eur J Surg Oncol. 2015;41(2):265-269.

34. He Z, Lu H, Du X, Hu W, Zhaoda BT. CA19-9 as a predictor of resectability in patients with borderline resectable pancreatic cancer. Hepatogastroenterology. 2013;60(124):900-903.

35. Kim YC, Kim HJ, Park JH, et al. Can preoperative CA19-9 and CEA levels predict the resectability of patients with pancreatic adenocarcinoma? J Gastroenterol Hepatol. 2009;24(12):1869-1875.

36. Turrini O, Schmidt CM, Moreno J, et al. Very high serum CA 19-9 levels: a contraindication to pancreaticoduodenectomy? J Gastrointest Surg. 2009;13(10):1791-1797.

37. Kondo N, Murakami Y, Uemura K, et al. Prognostic impact of perioperative serum CA 19-9 levels in patients with resectable pancreatic cancer. Ann Surg Oncol. 2010;17(9):2321-2329.

38. Hata S, Sakamoto Y, Yamamoto Y, et al. Prognostic impact of postoperative serum CA 19-9 levels in patients with resectable pancreatic cancer. Ann Surg Oncol. 2012;19(2):636-641.

39. Sugiura T, Uesaka K, Kanemoto H, et al. Serum CA19-9 is a significant predictor among preoperative parameters for early recurrence after resection of pancreatic adenocarcinoma. J Gastrointest Surg. 2012;16(5):977-985.

40. Katz MGH, Varadhachary GR, Fleming JB, et al. Serum Ca 19-9 as a marker of resectability and survival in patients with potentially resectable pancreatic cancer treated with neoadjuvant chemoradiation. Ann Surg Oncol. 2010;17(7):1794-1801.

41. Boone BA, Steve J, Zenati MS, et al. Serum CA 19-9 response to neoadjuvant therapy is associated with outcome in pancreatic adenocarcinoma. Ann Surg Oncol. 2014;21(13):4351-4358.

42. Williams JL, Kadera BE, Nguyen AH, et al. CA19-9 normalization during pre-operative treatment predicts longer survival for patients with locally progressed pancreatic cancer. J Gastrointest Surg. 2016; 20(7):1331-1342.

43. Berger AC, Meszoely IM, Ross EA, Watson JC, Hoffman P. Undetectable preoperative levels of serum CA 19-9 correlate with improved survival for patients with resectable pancreatic adenocarcinoma. Ann Surg Oncol. 2004;11(7):644-649.

44. Brown EG, Canter RJ, Bold RJ. Preoperative CA 19-9 kinetics as a prognostic variable in radiographically resectable pancreatic adenocarcinoma. J Surg Oncol. 2015;111(3):293-298.

45. Barton JG, Bois JP, Sarr MG, et al. Predictive and prognostic value of CA 19-9 in resected pancreatic adenocarcinoma. J Gastrointest Surg. 2009;13(11):2050-2058.
46. Goonetilleke KS, Siriwardena AK. Systematic review of carbohydrate antigen (CA 19-9) as a biochemical marker in the diagnosis of pancreatic cancer. Eur J Surg Oncol. 2007;33(3):266-270.

47. Wu L, Huang P, Wang F, et al. Relationship between serum CA 19-9 and CEA levels and prognosis of pancreatic cancer. Ann Transl Med. 2015;3(21):328

48. Lee KJ, Yi SW, Chung MJ, et al. Serum CA 19-9 and CEA levels as a prognostic factor in pancreatic adenocarcinoma. Yonsei Med $J$. 2013;54(3):643-649.

49. Schmidt C. CA-125: a biomarker put to the test. J Natl Cancer Inst. 2011;103(17):1290-1291.

50. Chen T, Zhang MG, Xu HX, Wang WQ, Liu L, Yu XJ. Preoperative serum CA125 levels predict the prognosis in hyperbilirubinemia patients with resectable pancreatic ductal adenocarcinoma. Medicine (Baltimore). 2015;94(19):e751.

51. Luo G, Xiao Z, Long J, et al. CA125 is superior to CA19-9 in predicting the resectability of pancreatic cancer. J Gastrointest Surg. 2013; 17(2):2092-2098.

52. Mackey JR, Mani RS, Selner M, et al. Functional nucleoside transporters are required for gemcitabine influx and manifestation of toxicity in cancer cell lines. Cancer Res. 1998;58(19):4349-4357.

53. Greenhalf W, Ghaneh P, Neoptolemos JP, et al. Pancreatic cancer hENT1 expression and survival from gemcitabine in patients from the ESPAC-3 trial. J Natl Cancer Inst. 2014;106(1):djt347.

54. Farrell JJ, Elsaleh H, Garcia M, et al. Human equilibrative nucleoside transporter 1 levels predict response to gemcitabine in patients with pancreatic cancer. Gastroenterology. 2009;136(1):187-195.

55. Ormanns S, Heinemann V, Raponi M, et al. Human equilibrative nucleoside transporter 1 is not predictive for gemcitabine efficacy in advanced pancreatic cancer: translational results from the AIO-PK0104 phase III study with the clone SP120 rabbit antibody. Eur J Cancer. 2014;50(11): 1891-1899.

56. Sinn M, Riess H, Sinn BV, et al. Human equilibrative nucleoside transporter 1 expression analysed by the clone SP 120 rabbit antibody is not predictive in patients with pancreatic cancer treated with adjuvant gemcitabine - results from the CONKO-001 trial. Eur J Cancer. 2015; 51(12):1546-1554.

57. Paproski RJ, Young JD, Cass CE. Predicting gemcitabine transport and toxicity in human pancreatic cancer cell lines with the positron emission tomography tracer 3'-deoxy-3'-fluorothymidine. Biochem Pharmacol. 2015;79(4):587-595.

58. Faca VM, Song KS, Wang H, et al. A mouse to human search for plasma proteome changes associated with pancreatic tumor development. PLoS Med. 2008;5(6):e123.

59. Poruk KE, Firpo MA, Scaife CL, et al. Serum osteopontin and tissue inhibitor of metalloproteinase 1 as diagnostic and prognostic biomarkers for pancreatic adenocarcinoma. Pancreas. 2013;42(2):193-197.

60. Mroczko B, Lukaszewicz-Zajac M, Wereszczynska-Siemiatkowska U, et al. Clinical significance of the measurements of serum matrix metalloproteinase-9 and its inhibitor (tissue inhibitor of metalloproteinase-1) in patients with pancreatic cancer: metalloproteinase- 9 as an independent prognostic factor. Pancreas. 2009;38(6):613-618.

61. Brand RE, Nolen BM, Zeh HJ, et al. Serum biomarker panels for the detection of pancreatic cancer. Clin Cancer Res. 2011;17(4):805-816.

62. Nolen BM, Brand RE, Prosser D, et al. Prediagnostic serum biomarkers as early detection tools for pancreatic cancer in a large prospective study. PLoS One. 2014;9(4):e94928.

63. Firpo MA, Boucher KM, Mulvihill SJ. Prospects for developing an accurate and diagnostic biomarker panel for lower prevalence cancers. Theor Biol Med Model. 2014;11:34.

64. Gerdtsson AS, Malats N, Sall A, et al. A multicenter trial defining a serum protein signature associated with pancreatic ductal adenocarcinoma. Int J Proteomics. 2015;2015:587250.

65. Gerdtsson AS, Wingren C, Persson H, et al. Plasma protein profiling in a stage defined pancreatic cancer cohort - implications for early diagnosis. Mol Oncol. 2016;10(8):1305-1316. 
66. Vasen H, Ibrahim I, Ponce CG, et al. Benefit of surveillance for pancreatic cancer in high-risk individuals: outcome of long-term prospective follow-up studies from three European expert centers. J Clin Oncol. 2016;34(17):2010-2019.

67. Petrushnko W, Gundara JS, De Reuver PR, O'Grady G, Samra JS, Mittal A. Systematic review of peri-operative prognostic biomarkers in pancreatic ductal adenocarcinoma. HPB (Oxford). 2016;18(8): 652-663.

68. Bartel DP. MicroRNAs: genomics, biogenesis, mechanism, and function. Cell. 2004;116(2):281-297.

69. Esquela-Kerscher A, Slack FJ. Oncomirs - microRNAs with a role in cancer. Nat Rev Cancer. 2006;6(4):259-269.

70. Jamieson NB, Morran DC, Morton JP, et al. MicroRNA molecular profiles associated with diagnosis, clinicopathologic criteria, and overall survival in patients with resectable pancreatic ductal adenocarcinoma. Clin Cancer Res. 2012;18(2):534-545.

71. Papaconstantinou IG, Manta A, Gazouli M, et al. Expression of microRNAs in patients with pancreatic cancer and its prognostic significance. Pancreas. 2013;42(1):67-71.

72. Papaconstantinou IG, Lykoudis PM, Gazouli M, Manta A, Polymeneas G, Voros D. A review on the role of microRNA in biology, diagnosis, and treatment of pancreatic adenocarcinoma. Pancreas. 2012; 41(5):671-677.

73. Vila-Navarro E, Vila-Casadesus M, Moreira L, et al. MicroRNAs for detection of pancreatic neoplasia: biomarker discovery by next-generation sequencing and validation in 2 independent cohorts. Ann Surg. Epub 2016 May 26.

74. Kamat AA, Baldwin M, Urbauer D, et al. Plasma cell-free DNA in ovarian cancer: an independent prognostic biomarker. Cancer. 2010; 116(8):1918-1925.

75. Diaz LA Jr, Bardelli A. Liquid biopsies: genotyping circulating tumor DNA. J Clin Oncol. 2014;32(6):579-586.

76. Hadano N, Murakami Y, Uemura K, et al. Prognostic value of circulating tumour DNA in patients undergoing curative resection for pancreatic cancer. Br J Cancer. 2016;115(1):59-65.

77. Tjensvoll K, Lapin M, Buhl T, et al. Clinical relevance of circulating KRAS mutated DNA in plasma from patients with advanced pancreatic cancer. Mol Oncol. 2016;10(4):635-643.

78. Cristofanilli M, Budd GT, Ellis MJ, et al. Circulating tumor cells, disease progression, and survival in metastatic breast cancer. $N$ Engl J Med. 2004;351(8):781-791.
79. Nagrath S, Sequist LV, Maheswaran S, et al. Isolation of rare circulating tumour cells in cancer patients by microchip technology. Nature. 2007;450(7173):1235-1239.

80. Vona G, Estepa L, Beroud C, et al. Impact of cytomorphological detection of circulating tumor cells in patients with liver cancer. Hepatology. 2004;39(3):792-797.

81. Hofman V, Long E, Ilie M, et al. Morphological analysis of circulating tumour cells in patients undergoing surgery for non-small cell lung carcinoma using the isolation by size of epithelial tumour cell (ISET) method. Cytopathology. 2012;23(1):30-38.

82. Kurihara T, Itoi T, Sofuni A, et al. Detection of circulating tumor cells in patients with pancreatic cancer: a preliminary result. J Hepatobiliary Pancreat Surg. 2008;15(2):189-195.

83. Tjensvoll K, Nordgard O, Smaaland R. Circulating tumor cells in pancreatic cancer patients: methods of detection and clinical implications. Int J Cancer. 2014;134(1):1-8.

84. Z'graggen K, Centeno BA, Fernandez-del Castillo C, Jimenez RE, Werner J, Warshaw AL. Biological implications or tumor cells in blood and bone marrow of pancreatic cancer patients. Surgery. 2001;129(5):537-546.

85. Soeth E, Gringoleit U, Moellmann B, et al. Detection of tumor cell dissemination in pancreatic ductal carcinoma patients by CK 20 RT-PCR indicates poorer survival. J Cancer Res Clin Oncol. 2005;131(10): 669-676.

86. de Albuqueruqe A, Kubisch I, Breier G, et al. Multimarker gene analysis of circulating tumor cells in pancreatic cancer patients: a feasibility study. Oncology. 2012;82(1):3-10.

87. Poruk KE, Valero V 3rd, Saunders T, et al. Circulating tumor cell phenotype predicts recurrence and survival in pancreatic adenocarcinoma. Ann Surg. Epub 2016 Jan 7.

88. Yang J, Weinber RA. Epithelial-mesenchymal transition: at the crossroads of development and tumor metastasis. Dev Cell. 2008;14(6):818-829.

89. Cauley CE, Pitman MB, Zhou J, et al. Circulating epithelial cells in patients with pancreatic lesions: clinical and pathologic findings. $J \mathrm{Am}$ Coll Surg. 2015;221(3):699-707.

90. Court CM, Ankeny JS, Hou S, Tseng HR, Tomlinson JS. Improving pancreatic cancer diagnosis using circulating tumor cells: prospects for staging and single-cell analysis. Expert Rev Mol Diagn. 2015;15(11): 1491-1150.
OncoTargets and Therapy

\section{Publish your work in this journal}

OncoTargets and Therapy is an international, peer-reviewed, open access journal focusing on the pathological basis of all cancers, potential targets for therapy and treatment protocols employed to improve the management of cancer patients. The journal also focuses on the impact of management programs and new therapeutic agents and protocols on

\section{Dovepress}

patient perspectives such as quality of life, adherence and satisfaction. The manuscript management system is completely online and includes a very quick and fair peer-review system, which is all easy to use. Visit http://www.dovepress.com/testimonials.php to read real quotes from published authors. 